\title{
Spectrum of Biopsy Proven Glomerular Disease in Children at Kanti Children's Hospital
}

\author{
Khatun $\mathbf{N}^{1}$, Bista KP², Mahaseth $\mathrm{C}^{3}$
}

\begin{abstract}
Introduction: Glomerular disease is one of the most common forms of renal disease and can have many different clinical presentations. However there is variation in the prevalence in the type of glomerular disease according to geographical location and race of population. The aim of the study was to find the overall distribution pattern of glomerular disease based on renal biopsies. Material and Methods: The medical records of all children who underwent kidney biopsy $(n=29)$ between January 2012-june 2014 were analyzed. In this retrospective study we review children from Kanti Children's Hospital, Nephrology Department. Demographic data including age, sex and indication of kidney biopsy as well as complication of the procedures were recorded. Result: A total number of thirty one biopsies were done. Two children were excluded from the study due to inadequate tissue and artifacts defects in preservative. The remaining twenty nine biopsies were included in the study and analyzed. Renal disease was found nearly equal in both male and female (51.72\% vs. $48.27 \%$ ). Mean age was $10.95 \pm 3.30$ years. Maximum number of biopsies $(75.86 \%)$ was performed between 10- 15 years. The most common indication of the kidney biopsy was nephrotic syndrome (steroid resistant nephrotic syndrome and steroid dependent nephrotic syndrome, 31.02\% followed by lupus nephritis $27.58 \%$.The most common glomerular disease were focal segmental glomerulosclerosis and lupus nephritis both contribute $27.58 \%$. Among lupus nephritis class III was found more common (44.44\%) in children. IgA nephropathy was also contribute (17.24\%) in all glomerular disease. Regarding the kidney biopsy complication gross hematuria was observed in $6.89 \%$ of children which was self-limited. Conclusion: FSGS and lupus nephritis is becoming the most common glomerular disease in children attending the Kanti Children Hospital.IgA nephropathy was also contribute the glomerular disease in the children.
\end{abstract}

Key words: Complication, Glomerulonephritis, Kidney biopsy, Nephrotic syndrome

\section{Introduction}

idney biopsy in the pediatric age group as in the adult population is one of the most important investigation in renal medicine.
'Dr. Najala Khatun, MBBS, MD, ${ }^{2}$ Dr. Krishna Prasad Bista, MBBS, DCH, ${ }^{3}$ Professor Dr. Chandeshwar Mahaseth, MBBS, DCH, MD, MRCPCH. All from Kanti Children's Hospital, Maharajgunj, Kathmandu, Nepal.

\author{
Address for correspondence: \\ Dr. Najala Khatun \\ Kanti Children's Hospital \\ Maharajgunj, Kathmandu, Nepal \\ Email: najala-khatun@hotmail.com \\ Tel: 977-9841785041
}

\section{How to cite}

Khatun N, Bista KP, Mahaseth C. Spectrum of Biopsy Proven Glomerular Disease in Children at Kanti Children's Hospital. J Nepal Paediatr Soc 2014;34(3):225-229.

doi: http://dx.doi.org/10.3126/jnps.v34i3.11162

This work is licensed under a Creative Commons Attribution 3.0 License.

(c) (7)

In children the rate of undergoing kidney biopsy is not more than $10 \%$ as compared to adult ${ }^{1}$. Since the introduction of percutaneous renal biopsy (PRB) in 1950's, technical advances in imaging and biopsy needle have simplified and improve the success of the procedures. Though PRB is generally considered a safe but it is more difficult in children than adults due to size variation and different level of cooperation $^{2,3,4}$. It can provide diagnostic precision, especially in glomerular disease, and also provides important information of prognostic value and about treatment option ${ }^{5}$. However, there is a variation in the prevalence of the types of glomerular 
disease according to geographical location and race of the population ${ }^{6}$. Glomerular disease is one of the most common forms of renal disease and can have many different clinical presentation. Glomerular diseases continue to be the leading causes of end stage renal disease globally, ${ }^{6,7}$. There is only few published data regarding the frequency of different glomerulonephritis in Nepalese children in renal biopsies. Therefore, this was conducted to observe the spectrum of glomerular disease in children attending the Kanti Children's Hospitals.

\section{Materials and Methods}

All the kidney biopsies that were performed in Kanti Children's Hospital, Nephrology Department over a period of two and half year from January 2012 to June 2014 were retrospectively analyzed. A total thirty one biopsies were done. Two cases of renal biopsies were excluded from the analysis due to inadequate tissue and artifacts defect in preservative. Finally twenty nine biopsies were analyzed.

Demographic data including age, sex, as well as indication of kidney biopsy, the histopathological diagnosis and complications of the procedure were recorded.

Biopsies were done under midazolam premedication, using Bard Disposable Core biopsy 18 G. Smaller children less than five years, biopsies were done under ultrasound guidance and children more than five years biopsies were done after ultrasound guidance surface marking. Two specimens were taken for light microscope and for immunohistochemistry. All our biopsies were done as an inpatient procedure and patients were observed for twenty four hours after the procedure.

Each biopsy specimen was stained with hemotoxyline and eosine ( $\mathrm{H}$ and $\mathrm{E})$, periodic acid Schiff (PAS), silver methenamine, congo red. IgA, IgM, IgG,
C3, C1q, kappa and lambda light chain were tested by direct immunofluoroscence.

Nephrotic syndrome was defined as clinical triad of heavy proteinuria ( 24 hours urine protein $40 \mathrm{mg} / \mathrm{m}^{2}$ / hour or $2 \mathrm{mg} / \mathrm{mg}$ ), hypoalbuminemia and generalized edema. Steroid resistant nephrotic syndrome was defined as persistent proteinuria of more than $40 \mathrm{mg} /$ $\mathrm{m} 2$ /hour after 8 weeks of daily dose of prednisolone therapy. Steroid dependent nephrotic syndrome is defined as relapse while on alternate day steroid therapy or within 21 days of completing a successful course of prednisolone therapy.

\section{Results}

Over two and half year period, thirty one biopsies were performed, and two cases were excluded. Finally twenty nine biopsies were analyzed. Where male $(n=14)$ and female $(n=15)$ were nearly equal. Mean age was $10.95( \pm 3.30)$ years, ranging from 22 months to 14 years. Among these $6.89 \%$ were aged under 5 years, $17.24 \%$ were $6-10$ years and $75.86 \%$ were $11-14$ years (Figure 1). FSGS, IgA nephropathy, lupus nephritis were more common between 10-14 years (Table 2).

The most common indication for renal biopsy was nephrotic syndrome $31.02 \%$, among SRNS and SDNS was $24.13 \%$ and $6.89 \%$ respectively. Suspected lupus nephritis was second most common indication for biopsy (Table 1).

The most common glomerular disease was focal segmental glomerulosclerosis (FSGS) and lupus nephritis (27.58\%). Among the lupus nephritis class III was the most common glomerular disease in children followed by class IV. IgA nephropathy was found $17.24 \%$ (Table 3). None of the biopsies shows minimal changes nephrotic syndrome. Following the procedures 6.89\% patients developed gross hematuria which was selflimited (Table 4).

Table 1: Indication for kidney biopsy:

\begin{tabular}{|c|c|c|}
\hline Indication & Number $(n=29)$ & Percentage (\%) \\
\hline \multicolumn{3}{|l|}{ Nephrotic syndrome } \\
\hline Steroid resistant nephrotic syndrome (SRNS) & 7 & 24.13 \\
\hline Seroid dependent nephrotic sundrome(SDNS) & 2 & 6.89 \\
\hline Recurrent gross hematuria & 2 & 6.89 \\
\hline Persistent hematuria & 0 & 0 \\
\hline Suspected lupus nephritis & 8 & 27.58 \\
\hline Henoch schönlein purpura with nephrotic range proteinuria & 2 & 6.89 \\
\hline Systemic disease with nephrotic range proteinuria & 1 & 3.44 \\
\hline Rapid deterioration of renal function & 2 & 6.89 \\
\hline Nephrotic range proteinuria with hematuria & 5 & 17 \\
\hline
\end{tabular}


Table 2: Histopathological diagnosis of children according to age category:

\begin{tabular}{|c|c|c|c|}
\hline & $0-4$ years & 5-9 years & $10-14$ years \\
\hline Minimal change nephrotic syndrome & 0 & 0 & 0 \\
\hline Focal segmental glomerulosclerosis & 1 & 2 & 5 \\
\hline Mesangiocapillary glomerulonephritis & 0 & 0 & 1 \\
\hline Diffuse proliferative glomerulonephritis & 0 & 1 & 1 \\
\hline IgA nephropathy & 0 & 0 & 5 \\
\hline Henoch schonlein glomerulonephritis & 0 & 0 & 2 \\
\hline Lupus nephritis & 0 & 1 & 7 \\
\hline Non immune complex mediated glomerulopathy & 0 & 1 & 0 \\
\hline Post infectious & 0 & 0 & 1 \\
\hline Non specific & 1 & 0 & 0 \\
\hline
\end{tabular}

Table 3: Distribution of Glomerular Diseases

\begin{tabular}{|l|c|c|}
\hline Glomerular Diseases & Number (n=29) & Percentage (\%) \\
\hline Minimal change nephrotic syndrome & 0 & 27.58 \\
\hline Focal segmental glomerulosclerosis & 1 & 3.44 \\
\hline Mesangiocapillary glomerulonephritis & 2 & 6.89 \\
\hline Diffuse proliferative glomerulonephritis & 5 & 17.24 \\
\hline IgA nephropathy & 2 & 6.89 \\
\hline Henoch Schönlein Purpura & 1 & 3.44 \\
\hline Post infectious glomerulonephritis & 8 & 27.58 \\
\hline Lupus nephritis & 0 & \\
\hline Class I & 0 & \\
\hline Class II & $4 / 8$ & \\
\hline Class III & $3 / 8$ & \\
\hline Class IV & 0 & \\
\hline Class V & $1 / 8$ & 3.44 \\
\hline Class III and V & 1 & 3.44 \\
\hline Non immune complex mediated glomerulopathy & 1 & \\
\hline Non specific & & \\
\hline
\end{tabular}

Table 4: Procedure Complication

\begin{tabular}{|l|c|c|}
\hline Biopsy Complication & Number $(\mathbf{n})$ & Percentage (\%) \\
\hline Microscopic hematuria & 0 & 6.89 \\
\hline Gross hematuria & 2 & 0 \\
\hline Perinephric hematoma & 0 & 0 \\
\hline AV fistula & 0 & 0 \\
\hline Infection at biopsy site & 0 & 0 \\
\hline
\end{tabular}




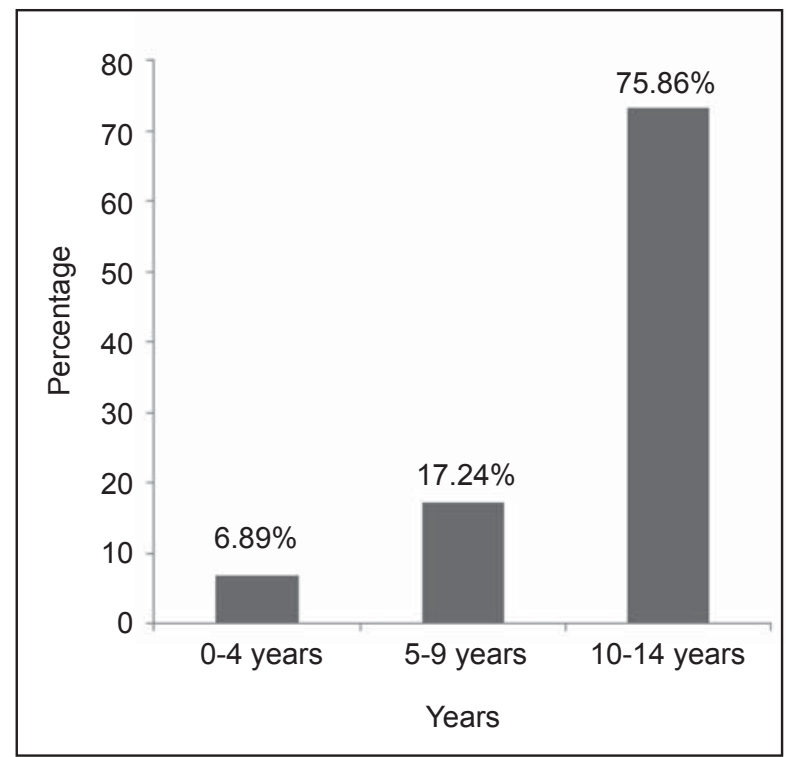

\section{Discussion}

The study provides the spectrum of glomerular disease in children attending the Nephrology Department at Kanti Children's Hospital. In the present study, biopsy was done nearly equal in male and female. Study from Jordan shows requirement of biopsy was more common in male but female were more in Australia ${ }^{1,6}$.

The maximum numbers $(75.86 \%)$ of biopsies are performed at 10-14 years of age. Printza et al. also mentioned maximum numbers of biopsies were performed at $11-14$ years $(48 \%)^{2}$. This is because clinically up to age 10 years we treated as minimal change nephrotic syndrome and biopsy was not regularly performed. The most common indication for biopsy at our center was nephrotic syndrome (SRNS and SDNS) followed by suspected lupus nephritis. Nephrotic syndrome was the most frequent indication for renal biopsy in United Kingdom (22.4\%), Serbia as well as in India ${ }^{4,6,9}$. In our country nephrotic syndrome was also common indication for renal biopsy (81.6\%) in adult ${ }^{12}$. Similar study done by Edward et al. shows nephrotic syndrome (SRNS and SDNS) was common indication for children and adult. However, SRNS and minimal changes nephrotic syndrome was common indication of biopsy shown by Pralad and Viswanath ${ }^{1,12,13}$.

In our study FSGS (27.58\%) and lupus nephritis $(27.58 \%)$ were the most common glomerular disease in children. The change in the spectrum of the glomerular diseases in the last few decades has been demonstrated in many studied worldwide with most showing a trend towards increase in FSGS cases $^{8}$. Also in adult population
FSGS is more common in India (80\%), Nepal (81.6\%), Bangladesh $(20.41 \%)^{6,10,11}$. Various study shown that minimal changes disease (MCD) are the most common form of nephrotic syndrome in children ${ }^{12}$. However this could be explained by the fact that those children with clinical and laboratory evidences of MCD were not subjected to kidney biopsy. Also in our study none of the children with MCD were included for renal biopsy. Golay et al. mentioned lupus nephritis was most common secondary glomerulonephritis (73.38\%) and uniformly the commonest causes of of secondary glomerulonephritis worldwide ${ }^{7}$.

In our study among $27.58 \%$ of lupus nephritis, class III corresponds to $44.44 \%$ followed by class IV but it was more common in eastern Nepal ${ }^{13}$. This variation is due to different geographical region and race of the population. In the present study, IgA nephropathy was found $17.24 \%$ in studied children but this is not consistent with the study done at Dharan, where IgA nephropathy was not reported. Golay et al. also mentioned prevalence of IgA nephropathy is much lower in Nepal ${ }^{7,11}$. This discrepancy may be due to fact that Kanti Children's Hospital is only pediatrics tertiary referral hospital and lack of published data in Nepal. Report from the Italian national registry of renal biopsies in children idiopathic IgA nephropathy was found most common (18.8\%) $)^{9}$. Among the adult population IgA nephropathy was found $2.9 \%$ in Nepal ${ }^{11}$.

Following the procedure $6.89 \%$ children has developed gross hematuria. Our study is not consistent with the study done at Jordan (13.8\%) and India $(16.8 \%)$, which was nearly two times higher than our study ${ }^{1,14}$. This difference is may be due to simplified biopsy needle and ultra sound guidance biopsy in younger children. William et al. mentioned gross hematuria occurs in $3.1 \%$ in adult $^{3}$.

\section{Conclusion}

The most common indication for the renal biopsy is nephrotic syndrome and suspected lupus nephritis. FSGS and lupus nephritis is becoming the most common glomerular disease in children attending the Kanti Children's Hospital. IgA nephropathy is also contributing the glomerular disease in children. The procedure is proved to be safe.

\section{Recommendation}

National registries for paediatric kidney biopsy should be established from hospitals of different region of Nepal. 
Acknowledgement: We would like to thank, Dr. Hensen Khadka, Dr. Prasant Khatiwada, and Dr. Bhawani Yadav, radiologist in Kanti Children's Hospital who helps us in ultrasound guided kidney biopsy and surface marking.

Funding: Nil

Conflict of Interest: None

Permission from IRB: Yes

\section{Reference}

1. Edward S, Issa Haja, Ola El I, Mona K. Spectrum of biopsy proven renal disease in the paediatric age group at king hussain medical center. JRMS 2007;14(1):34-37.

2. Prinza N, Bosdou J, Panjaki A, Badouraki M, Kollios K, Glogha C. Percutaneous ultrasound guided renal biopsy in children: a single centre experience. Hippokratia 2011;15(3)258:261.

3. Willian $L$, tephen $\mathrm{N}$,. Timing of complication in percutaneous renal biopsy. I Am Soc Nephrol 2004;15:142-47

4. Farida H, Meeta M, Stephen D,Alam WR. Renal biopsy in children: current practice and audit of outcomes. Nephrol D Transplant 2010; 25:485-89.

5. Yuen LK, Lai WM, Lau SC, Tong PC, Tse KC, Chiu $M C$. Ten years review of disease pattern from percutaneous renal biopsy: an experience from a Paediatric tertiary renal centre in Hong Kong. Hong Kong Med J 2008; 14:348-55.

6. Esther MB, John D, Moira F et al. The incidence of biopsy proven glomerulonephritis in Australia. Nephrol Dial Transplant 2001;16:1364-367.
7. Goyal V, Trivedi M, Abraham A, Roychowdhary A, Panday R. The spectrum of glomerular disease in single center: Clinicopathlogical correlation. Indian J Nephrol 2013;23(3):168-75.

8. Paripovic $D$, Kostic $M$, Kruscic $D$ et al. Indications and results of renal biopsy in children: a ten year review from a single center in Serbia. Societa Italiana dis Nefrologia 2012;225(06):1054-1059.

9. Cuppo R,Gianoglio B,Porcellini MG.Frequency of renal disease and clinical indication of renal biopsy in children ( Report of the Italian National Registry of Renal Biopsy in children. Nephrol Dial Transplant 1998;13:293-97.

10. Hossain MT, Begam M, Rahman AJE, Kamal M. Immmune deposits in glomerular disease and their and immunopathlogical correlation. Bangladesh J Pathol 2011;26(1):14.

11. Budhathoki S, Shrestha P, Bhatta N, Singh R et al. Systemic lupus erythematous in childhood- a review of 11 patients at a single center in eastern Nepal. Nepal Med Coll J 2007;3(3):186:190.

12. Aryal G. Histopathological spectrum of glomerular disease in Nepal: a seven year retrospective study. Nepal Med Coll J 2008; 10(2):126-128.

13. Shrestha $P$, Bhatta NK, Tiwari A, Singh R. Glomerular diseases in children- A review of 27 cases recorded at single center in Eastern Nepal. J Nepal Pediatric Society 2008; 29(1):26-29.

14. Pralad V, Vishwanath S. Renal biopsy: Procedure. Principles and practice of Pediatric Nephrology. Second edition. Jaypee, 2013; 169-174. 